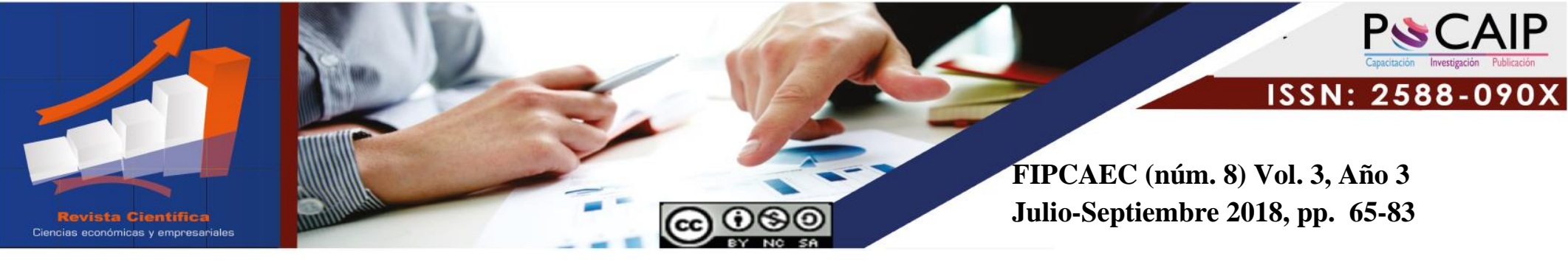

DOI: $10.23857 /$ fipcaec.v3i8.58

\title{
Gestión de recursos humanos en la empresa: un paradigma del siglo XXI
}

\section{Human resources management in the company: a paradigm of the 21 st century}

\section{Gestão de recursos humanos na empresa: um paradigma do século XXI}

\author{
Víctor Reinaldo Jama-Zambrano ${ }^{1}$ \\ victorreinaldojamas@hotmail.com \\ https://orcid.org/0000-0001-7102-2352
}

Correspondencia: victorreinaldojamas@ hotmail.com

* Recepción: 11/ 04/ 2018 *Aceptación: 12/06/ 2018 *Publicación: 15 /07/ 2018

Doctor en Ciencias de la Educación Especialización Pedagogía, Especialista en Desarrollo de la Inteligencia y Educación, Magíster en Educación y Desarrollo Social, Diploma Superior en Educación Universitaria por Competencias, Licenciado en Ciencias de la Educación en la Especialidad de Contabilidad y Administración, Profesor de Segunda Enseñanza en la Especialidad de Contabilidad y Administración, Profesor de Educación Primaria - Nivel Tecnológico, Docente del Instituto Superior Pedagógico Eugenio Espejo, Chone, Ecuador. 


\title{
Resumen
}

El conjunto de políticas y prácticas necesarias para administrar todos los aspectos relacionados con las personas se denomina gestión del talento humano, abarcando reclutamiento, selección, capacitación, recompensas, evaluación del desempeño, remuneración y calidad de vida. El capital humano con la globalización se ha transformado en el eslabón vital para el desarrollo empresarial. La gestión del talento humano tiene diversas orientaciones. Entre las funciones se hallan la planificación de necesidades de personal, motivación laboral, comunicación interna etc. el mismo se preside, orienta y se evalúa por indicadores y estrategias. El valor de la gestión de recursos humanos reside en que las empresas deben dar respuestas a los cambios en la sociedad en general, en la mentalidad de las nuevas generaciones que llegan a chocar con las generaciones anteriores y a los cambios en los modelos laborales que buscan darles más libertad a los empleados. El éxito empresarial depende de una adecuada gestión del talento humano.

Palabras clave: Gestión; recursos humanos; empresariales.

\begin{abstract}
The set of policies and practices necessary to manage all aspects related to people is called human talent management, including recruitment, selection, training, rewards, performance evaluation, remuneration and quality of life. Human capital with globalization has become the vital link for business development. Human talent management has different orientations. Among the functions are the planning of personnel needs, work motivation, internal communication etc. It is chaired, guided and evaluated by indicators and strategies. The value of human resources management lies in the fact that companies must respond to changes in society in general, in the mentality of the new generations that come to collide with previous generations and the changes in the labor models that seek to give them More freedom for employees. Business success depends on proper management of human talent.
\end{abstract}

Keywords: Management; human Resources; business. 


\section{Resumo}

$\mathrm{O}$ conjunto de políticas e práticas necessárias para gerenciar todos os aspectos relacionados às pessoas é chamado de gestão de talentos humanos, incluindo recrutamento, seleção, treinamento, recompensas, avaliação de desempenho, remuneração e qualidade de vida. O capital humano com a globalização se tornou o elo vital para o desenvolvimento de negócios. A gestão de talentos humanos tem orientações diferentes. Entre as funções estão o planejamento de necessidades de pessoal, motivação para o trabalho, comunicação interna etc. É presidido, guiado e avaliado por indicadores e estratégias. O valor da gestão de recursos humanos reside no fato de que as empresas devem responder às mudanças na sociedade em geral, na mentalidade das novas gerações que passam a colidir com as gerações anteriores e nas mudanças nos modelos de trabalho que buscam dar a elas Mais liberdade para os funcionários. O sucesso nos negócios depende do gerenciamento adequado do talento humano.

Palavras-chave: Gestão; recursos humanos; negócios.

\section{Introducción}

En el variable mundo de hoy cada momento se amplifica, la certeza de que las organizaciones de triunfo son aquellas que consideran el talento humano como el recurso más apreciable que poseen y saben que sólo a través de él, se llega a la innovación y al desarrollo de ventajas competitivas.

En el orden de las ideas anteriores este escenario ceñido por el desarrollo de las fuerzas productivas, las interconexiones del mundo real reproducidas por el perfeccionamiento de la informática y las comunicaciones, han llevado al desarrollo del concepto de un fenómeno nuevo, el cual conocemos como Globalización. Resulta revelador el rol de las personas involucradas en proceso productivo, graficado en el movimiento de la sociedad industrial a la sociedad de la informatización y de ésta, a la sociedad del conocimiento. (Alonso, Parra, \& Arronte, 2011)

Prestigiosos investigadores expresan que el surgimiento de la gestión del capital humano fue en la Escuela Conductista, aseveraban Barnard y Lewin los cuales fueron sus principales representantes, la necesidad de afrontar los conocimientos de una manera interdisciplinaria para una dirección eficiente de las personas en las organizaciones, tal aseveración da cuenta de la 
importancia del sujeto en los procesos organizacionales encuadrado en Pragmatismo la cual se concentra en ampliar una relación interactiva de los seres humanos y el mundo a través de las acciones y la experiencia humana. (Del Canto, 2011)

Refiere Chiavenato que la gestión del talento humano es el conjunto de políticas y prácticas necesarias para dirigir todos los aspectos relacionados con las personas, contenido el reclutamiento, selección, capacitación, recompensas, evaluación del desempeño, remuneración y calidad de vida. La cultura de la empresa, la estructura asimilada por la organización, las características del contexto ambiental, el negocio de la organización, la tecnología utilizada y los procesos internos son los elementos primordiales de la gestión del talento humano. Vale la pena plantear que la gestión del talento humano está conformada por las personas y las organizaciones, por lo cual, es básico el trato que se les da a las personas, sea como socios o como recursos.

Así mismo otros estudioso como García y Sabater conceptualizan la gestión del talento humano como un conjunto de actividades y medidas concretas, patrocinadas por los dirigentes en línea de la empresa, o encargados de determinadas funciones dentro del departamento de recursos humanos, teniendo un efecto directo sobre los comportamientos, actitudes, satisfacción de una persona o un grupo reducido de personas dentro de la organización (Del Canto, 2011).

En ese mismo orden de ideas Quintanilla define que la gestión del talento humano está orientada a la conformación y optimación del comportamiento de las personas, desde las expectativas de las organizaciones, pero además hay que tomar en cuenta las aspiraciones de cada uno de los empleados poseen, delimitan y caracterizan. (León, 2013)

El concepto de gerencia de talentos surge en siglo XXI por la necesidad de las organizaciones de salvaguardar el conocimiento gerenciando con asertividad al capital humano, como factor clave del éxito para el logro en su capital humano como fuente de creación el cual se convierta en mejora continua en las áreas estratégicas de la organización. (Del Canto, 2011)

En la extensión del tiempo mayormente se refleja en el ambiente gran competencia, cambios rápidos y permanentes, la supervivencia de una organización depende de su capacidad para generar valor agregado a sus clientes y proveedores, lo cual será la base de su competitividad y posicionamiento en el mercado, esto, estriba de la capacidad de innovación, velocidad de 
respuesta, adaptabilidad al cambio y habilidad para detectar las necesidades de los participantes en la cadena de valor de la organización; Constituye el capital humano un activo intangible que tiene la capacidad de apuntalar el aumento de la productividad, avivar la innovación y con ello la competitividad. Su hábitat le da características definitivamente particulares de cualquier otro tipo de recurso. Se puede afirmar que el triunfo de una organización se supedita apretadamente al desempeño de todas las personas que la forman, sin tener en cuenta su jerarquía, ubicación y condición ${ }^{4}$. De ahí la importancia de esta temática.

\section{Desarrollo (Valencia, 2005).}

La investigación del psicólogo australiano, profesor de la Universidad de Harvard, Elton Mayo y sus colaboradores, con una serie de estudios llevados a cabo en la planta de Hawthorne de la Western Electric Company (Estados Unidos), entre 1927 y 19321 se inició el movimiento de las relaciones humanas en la industria. Y el descubrimiento de la importancia del factor humano. Estas investigaciones se extendieron hasta 1932, las cuales facilitaron un escenario atrayente de la transición de la administración científica hasta el naciente movimiento de las relaciones humanas, representado por el énfasis en la conducta informal de los grupos de trabajo y en los sentimientos, actitudes e interacciones de los trabajadores.

A partir de estos acontecimientos se comienza, a prestársele atención al "recurso humano" en respuesta a la concepción del ser humano como un instrumento del proceso productivo, el individuo no es visto como algo "contra" lo cual hay que trabajar, sino como medio "con" el cual se enriquece el proceso.

En los momentos actuales resulta esencial plantear que el principal producto de las organizaciones.es el desarrollo humano. Se ha categorizado que el trabajador debe ser tratado como el cliente interno a quien hay que atenderle sus necesidades y expectativas legítimas para el logro de los cambios culturales y estructurales que favorezcan el mejoramiento de la calidad de vida de las personas y el desarrollo de la empresa, con la finalidad de mantener altamente la competitividad con base en la productividad. 
Listamos los tres enfoques que describió el Ministerio de Salud en 1999 relacionado con la importancia del recurso humano

Primer Enfoque: Crecimiento y Desarrollo de las Organizaciones. (Valentín, 2016)

El siglo XX será resonado, por el surgimiento de un número cada vez más creciente de organizaciones que con sus productos (bienes o servicios) han favorecido a que los seres humanos tengan un mejor nivel de vida.

Ejemplificamos: el campo de la medicina en los próximos años se trate enfermedades que hasta hoy son incurables. Este crecimiento y desarrollo de las organizaciones, será un crecimiento planeado, con visión de futuro, donde se integren todos los recursos y en el cual el factor humano se constituye como el principal componente.

\section{Segundo Enfoque: Búsqueda de la Excelencia en las Organizaciones}

Edward Deming fue el creador de enfoque, personaje importante dentro de la concepción de la filosofía de la calidad total. Deming, alegaba que si todos los trabajadores, desde su puesto de labores, se preocuparan por mejorar permanentemente la calidad de sus trabajos, aumentando la cantidad y reduciendo los costos asociados a los mismos; contribuyendo a que la organización sea vista como una entidad de reputación y competitiva en el mercado; cuando se logra establecer adecuadamente la calidad total en las organizaciones, promete un espacio ideal para el desarrollo, en búsqueda de la excelencia. Se proyecta, la calidad total en un enfoque de gestión de recursos humanos, que categóricamente obliga a ser constante en el proceso de cambio y mejoramiento continuo, debiendo implicar a todas las personas sin excepción que conforman la organización, incluido a los proveedores y clientes.

\section{Tercer Enfoque: La Globalización}

Se marca en este enfoque que las organizaciones modernas se identifican por ser: Globalizado, Competitivo, Cambiante (Constantemente varían las necesidades del cliente o la competencia mejora los beneficios de los productos/servicios). Los mercados globalizados, reclaman a las organizaciones que cumplan con cinco condiciones básicas para ser consideradas organizaciones competitivas: Los productos/servicios deben ser de alta calidad, deben ser proporcionados en la 
cantidad requerida, entregados en la oportunidad debida, los precios deben ser justos o razonables en comparación con los beneficios que brindan al cliente y deben ser entregados con una excelencia en el servicio.

\section{En la contemporaneidad es de observación obligada el conocimiento de las Características del Talento Humano}

En la actualidad el talento contiene cuatro aspectos esenciales para la competencia individual (Montoya \& Boyero, 2016)

- Conocimiento. Instituye el resultado de aprender a aprender, de forma continua, dado que el conocimiento es la moneda más valiosa del siglo XXI. ${ }^{6}$ (saber)

- Habilidad. Representa manejar y aplicar el conocimiento, ya sea para resolver problemas o situaciones, crear e innovar. En otras palabras, habilidad es la transformación del conocimiento en resultado. (saber hacer)

- Juicio. Analizar la situación y el contexto. Figura saber obtener datos e información, tener espíritu crítico, juzgar los hechos, ponderar con equilibrio y definir prioridades (Montoya \& Boyero, 2016).

- Actitud. La cualidad emprendedora permite alcanzar y superar metas, obtener riesgos, actuar como agente de cambio, agregar valor, llegar a la excelencia y enfocarse en los resultados, traslada a la persona a alcanzar la autorrealización de su potencial. (Montoya \& Boyero, 2016)

\section{Características de la Gestión del Talento Humano}

Según Chiavenato, los grandes cambios que se manifiestan en los escenarios económicos del presente conllevan a, que cada vez es más necesario comprender, aquello que influye sobre el rendimiento de los individuos en el trabajo, sobre su productividad, de cuáles deben ser los conocimientos que se deben manejar, en pro de lograr resultados positivos. En el ámbito Institucional se debe buscar nuevos métodos para captar y potenciar los recursos humanos, 
orientadas a encaminar en forma decisiva la dirección de una Institución, hasta conseguir el éxito deseado. La buena dirección de recursos humanos, ayuda a los colaboradores a utilizar su capacidad al máximo y a obtener no solo, satisfacción sino su integración como parte de un grupo de trabajo. (Montoya \& Boyero, 2016)

\section{Objetivos de la gestión de los recursos humanos (Reyes, 2015)}

La finalidad de la gestión de los recursos humanos debe contribuir con que la empresa produzca más y sea la mejor en fiel cumplimiento de estrategia de negocio. Se explica entonces que la gestión de los recursos humanos busca mejorar el rendimiento del trabajo, brindar bienestar a los empleados y explicar claramente las tareas a desempeñarse.

\section{Objetivos Explícitos}

La gestión eficiente de los recursos humanos se logra con el cumplimiento de cuatro objetivos explícitos primordiales

- Cautivar candidatos potencialmente calificados y capaces de desarrollar o adquirir las competencias necesarias de la empresa u organización.

- Detener a los empleados deseables

- Engendrar a los empleados para adquieran un compromiso con la empresa y se involucren en ella.

- Auxiliar a los empleados a crecer y desarrollarse dentro de la empresa.

\section{Objetivos implícitos}

Relacionamos los objetivos implícitos con los recursos humanos: mejora de la productividad, mejora de la calidad de vida en el trabajo y el cumplimiento de la normativa ${ }^{7}$.

- Productividad

- Calidad de vida en el trabajo

- Cumplimiento de la normativa

- Objetivos a largo plazo 
Es necesidad imperiosa estimar que la Gestión del Talento Humano moderna va más allá de la Administración de las Personas, está encaminada a la Gestión o Administración con las Personas. Se plantea como reto y desafío: lograr que las personas se sientan y actúen como socias de la organización, participen activamente en un proceso de desarrollo continuo a nivel personal y organizacional y sean los protagonistas del cambio y las mejoras.

Según Bolaño A (2015), el desarrollo del nuevo rol que asume RRHH, los profesionales de RRHH o aquellos que desarrollan tal función, requieren desarrollar cinco competencias claves

Conocimiento de la Organización: Concebir los indicadores clave del éxito organizacional que ilustren las relaciones entre la inversión de RRHH y el impacto estratégico en la organización. La victoria de RRHH no se mide mediante sus actividades, sino en los resultados organizacionales. Cuando a los directivos de las organizaciones se les especifica qué prácticas de RRHH podrían mejorar la calidad de la gestión y qué resultados podrían esperarse, se muestran mucho más favorables a invertir en RRHH

Puesta en marcha de prácticas de Recursos Humanos: Ser capaz de identificar qué prácticas ofrecen una mayor rentabilidad respecto a la inversión, cuáles tienen el mayor impacto estratégico o influyen más en los trabajadores.

Gestión cultural: Los profesionales de RRHH serían los llamados a trabajar en equipo con las áreas críticas y diseñar la mejora de los procesos. Asimismo, podría considerarse la participación de los usuarios en los procesos de mejora, tanto en el diagnóstico, como en las sugerencias y el diseño de las mejoras.

Gestión del cambio: Se deben entender los factores que intervienen y la situación específica de sus organizaciones para poder responder de manera apropiada a las demandas y problemas que surjan en el camino. 
Credibilidad personal: Serán cada vez más apreciados las personas que posean una competencia de gestión estratégica del conocimiento.

Procesos de la Gestión del Talento Humano (Rojas \& Vilchez, 2018)

Procesos para integrar personas. Se pueden citar procesos para proveer o abastecer personas. Contienen el reclutamiento y la selección de personal. Son los métodos para incluir a nuevas personas en la empresa.

Procesos para organizar a las personas. Métodos para diseñar las actividades que las personas realizarán en la empresa, para orientar y acompañar su desempeño. Envuelven el diseño organizacional y de puestos, el análisis y la descripción de los mismos, la colocación de las personas y la evaluación del desempeño.

Procesos para recompensar a las personas. Son los procesos para incitar a las personas y para satisfacer sus necesidades individuales más elevadas. Incluyen recompensas, remuneración y prestaciones y servicios sociales.

Procesos para desarrollar a las personas. Son los procesos para capacitar e incrementar el desarrollo profesional y personal. Implican la formación y el desarrollo, la administración del conocimiento y de las competencias, el aprendizaje, los programas de cambios y el desarrollo de carreras, y los programas de comunicación y conformidad.

- Procesos para retener a las personas. Es la creación de las condiciones ambientales y psicológicas satisfactorias para las actividades de las personas. Incluyen la administración de la cultura organizacional, el clima, la disciplina, la higiene, la seguridad y la calidad de vida y las relaciones sindicales. (Luna, 2012)

Procesos para auditar a las personas. Seguimiento y control de las actividades de las personas para verificar los resultados. Incluyen bancos de datos y sistemas de información administrativa. 


\section{Indicadores de la Gestión del Talento Humano}

\section{Proceso de Incorporación}

Se puede comentar que el proceso de incorporación es un filtro que permite ingresar a la organización a algunas personas, aquellas que cuentan con las características deseadas. En definitiva, la incorporación busca, de entre los diversos candidatos, a quienes sean más adecuados para los puestos de la organización o para las competencias que necesita; por tanto este proceso, pretende mantener o aumentar la eficiencia y el desempeño humano, así como la eficacia de la organización. De lo contrario, lo que está en juego es el capital intelectual que la organización debe preservar o enriquecer (Luna, 2012)

\section{Capacitación}

Es de esmerada importancia la capacitación la cual tiene como objetivo el mejoramiento de las competencias de las personas y, en consecuencia, el desempeño de la organización. Es uno de los métodos procesos más importantes de la administración de recursos humanos. La capacitación se diseña con el fin de proveer a los talentos el conocimiento y las habilidades que necesitan para el buen desempeño. (Castillo, 2012)

\section{Evaluación}

La evaluación del desempeño es una valoración, sistemática, de la actuación de cada persona en función de las actividades que desempeña, las metas y los resultados que debe alcanzar, las competencias que ofrece y su potencial de desarrollo. Es un proceso que sirve para juzgar o estimar el valor, la excelencia y las competencias de una persona, pero, sobre todo, la aportación que hace al negocio de la organización. (Castillo, 2012)

\section{Incentivos}

Una recompensa o incentivo es una gratificación, tangible o intangible, a cambio de la cual las personas asumen su decisión de participar en la organización y, una vez en ella, aportan su tiempo, esfuerzo y otros recursos personales (Castillo, 2012). 


\section{Funciones de la Gestión del Talento Humano}

\section{La planificación de necesidades de personal}

Indudablemente, ascender al máximo el potencial de las personas dentro de una empresa, es necesario saber qué tan necesitada está la empresa de ellas. La planificación es parte importante dentro de la misma planificación de la empresa, que tiene la función de asignar el número de trabajadores que se necesitan y que deben de tomarse según las capacidades de las mismas, sus deseos, que en los puestos asignados puedan desarrollarse, se mantengan motivados y así poseer un trabajo económicamente rentable (León, 2013)

\section{Análisis y descripción de puestos de trabajo}

Se define el Análisis de puesto como el proceso sistemático que consiste en determinar las habilidades, los deberes y conocimientos requeridos para desempeñar trabajos específicos en una empresa. Además, suministra información sobre la relación que tienen los trabajadores, las jerarquías y las condiciones en las que se realiza el trabajo. (León, 2013)

Administración de compensaciones (Hernández, Salazar, Rodríguez, \& S, 2010)

Esta función de la administración del recurso humano abarca, entre otros aspectos, la valoración del puesto, este es un procedimiento que proporciona información sobre el valor específico que un puesto determinado tiene, razón fundamental a la hora de constituir retribuciones. Para hacer una valoración de puestos se necesita la información aportada por el análisis y descripción de puestos.

\section{Reclutamiento y selección de personal}

Representa dos procesos principales dentro de una empresa, aunque en cierta forma se trabajan de manera separada, la ejecución de uno lleva al otro.

\section{Inducción de Personal}

Este programa es, de cierta forma, un plan sistemático para familiarizar al nuevo trabajador con la empresa (conocer sus áreas y departamentos) con los demás trabajadores (quienes serán sus jefes 
y subordinados y compañeros de trabajo) y con su puesto del trabajo (funciones y atribuciones) (Jimenez, 2018).

\section{Capacitación y Desarrollo}

Es de suma importancia el desarrollo de los recursos humanos -DRH- personifica una función, consistente en la capacitación, actividades de planeación y desarrollo de carreras individuales, desarrollo organización y evaluación del desempeño. La Capacitación y Desarrollo es el centro de un esfuerzo permanente de una organización, para diseñar mejoraras en las capacidades de los empleados y el desempeño de la empresa. (Jimenez, 2018)

\section{Plan de desarrollo de carrera profesional}

La planeación de una carrera no es solamente adquirir los conocimientos y habilidades de un puesto específico, sin dudas, que es primordial, pero además se deben de desarrollar otras habilidades para que el empleado tenga éxito. (Hernández, Salazar, Rodríguez, \& S, 2010)

\section{Evaluación del desempeño}

Es una apreciación metódica la evaluación del desempeño del trabajador o del potencial a desarrollar en el futuro, por lo que es necesario un proceso para estimular o calificar el valor de las cualidades de la persona. Este mismo es dinámico, efectuándose periódicamente a los empleados, ya sea este de manera formal o informal.

\section{Comunicación interna}

Una empresa tiene distintas maneras de enviar mensajes al exterior, al crear empleo, despido de trabajadores, por pago de salarios, sean estos elevados o bajo, en sí, esta cultura organizativa, se da a conocer en la calle. Por lo cual, uno de los elementos clave para que éste se comunique lo constituyen las personas que trabajan en la empresa. (Hernández, Salazar, Rodríguez, \& S, 2010) 


\section{Motivación laboral}

La motivación puede ser externa cuando se da desde fuera de la persona o interna cuando se da en el interior, es decir se motiva así mismo. Si se observa cualquier empresa y las personas que ocupan un mismo puesto y con las mismas condiciones de trabajo, en cuanto a su rendimiento existen diferencias. Las empresas deben de cuestionar porqué sucede esto y procurar comprender el comportamiento de los trabajadores, de este modo, utilizar un método de motivación para que sus miembros colaboren y cooperen en alcanzar las metas, de cierta forma, animándoles a compartir sus ideas y que realicen con entusiasmo el trabajo (Jimenez, 2018).

\section{Dirección de equipos de trabajo}

Numerosos administradores, piensan que deben controlar todo lo que sucede dentro de la empresa y tienen miedo a otorgar poder a sus empleados para que tomen decisiones por sí mismos (Jimenez, 2018).

Importancia de la Gestión de Recurso humano (Los Recursos Humanos, 2019).

Es vital la importancia del capital humano en las organizaciones. Por más que las máquinas haya reemplazado a los trabajadores en las fábricas aún se necesita del hombre para diseñar y fabricar las máquinas.

En el orden de las ideas anteriores es importante saber que las máquinas (los ordenadores o robots) no pueden reemplazar la creatividad, la emoción y muchas funciones que realiza el cerebro humano. Las relaciones interpersonales que acrecientan los negocios y las posibilidades de mejora y crecimiento tampoco pueden ser reemplazadas por las máquinas.

Cabe agregar que lo esencial del capital humano está en desarrollar una gestión de recursos humanos clave que cambie al conocimiento y habilidades de la gente en posibilidades concretas de consecución de objetivos.

Medina A. investigador del tema hace referencia de que el área de recursos humanos es muy importante no sólo para el reclutamiento del mejor equipo, sino también para la adaptación y capacitación de los miembros de la organización. El rendimiento de los trabajadores se lograra en la medida que estén más compenetrados con los objetivos y valores de la empresa, se deduce que 
la gestión de recursos humanos no debe limitarse a labores administrativas, sino que debe procurar crear un clima laboral agradable que genere un sentido de pertenencia y compromiso entre todos los trabajadores (Big River, 2018).

La parte más importante para el funcionamiento eficiente de una empresa es y siempre será su personal, que contribuye no sólo realizando las actividades necesarias para el funcionamiento del negocio, también aporta ideas, innovaciones y parte de su esencia y personalidad en los productos y servicios. Por tal motivo, la gestión de recursos humanos ha ganado mucha importancia en las últimas décadas, desde que se empezó a valorar la importancia del personal y sus aportaciones. (Big River, 2018)

La importancia de la gestión de recursos humanos en la actualidad reside en que las empresas, deben dar respuestas a los cambios en la sociedad en general, en la mentalidad de las nuevas generaciones que llegan a chocar con las generaciones anteriores y a los cambios en los modelos laborales que buscan darles más libertad a los empleados. Ente los cambios que más inciden y perturban a nivel laboral son (Los Recursos Humanos, 2019)

- Acrecentamiento de la competencia en el mercado general, y por ende la necesidad de ser competitivo.

- Los costos y ventajas relacionadas con el uso de los recursos humanos

- La crisis de productividad que afecta al mercado laboral

- El acrecimiento del ritmo y complejidad de los cambios sociales y el estilo de vida moderno. (Los Recursos Humanos, 2019)

Una apropiada respuesta a estos cambios logra que las compañías tengan ventajas competitivas, que las hacen destacar de otras empresas de su ramo, entre las principales podemos destacar

- Mejoran la eficiencia y eficacia. Cuando los trabajadores se sienten a gusto y cuentan con los medios para desarrollarse libremente, encuentran medios para disminuir los costos y proporcionan una atención única a los clientes. ${ }^{15}$

- Una adecuada gestión de RR HH les permitirá descubrir al talento de su organización, el cual sirve de ejemplo para que los demás imiten sus capacidades y contribuciones. 
- Sus talentos pueden combinarse y desplegarse para trabajar en nuevas tareas en cuanto les sea necesario, de forma organizada y eficiente, puesto que cada uno conoce su rol en la compañía. (Big River, 2018)

\section{Referencias}

1. Alonso, C., Parra, R., \& Arronte, L. (2011). Un sistema de gestión del capital humano en la emce basado en las competencias laborales. Rev Ing Ind, XXXI (1). Obtenido de http://www.redalyc.org/pdf/3604/360433572003.pdf\&sa=U\&ved=2ahUKEwiE3on

2. Big River. (12 de Enero de 2018). La importancia del capital humano para el éxito en los negocios. Obtenido de Sistema Recursos Humanos: http://sistemarecursoshumanos.com/la-importancia-los-objetivos-la-gestion-recursoshumanos/

3. Castillo, R. (2012). Desarrollo del capital humano en las organizaciones. . México: Red del Tercer Milenio. Obtenido de http://www.aliat.org.mx/BibliotecasDigitales/economico_administrativo/Desarrollo_del_c apital_humano_en_las_org.pdf

4. Del Canto, E. (2011). Gestión del capital humano, competencias y sociedad del conocimiento. Observatorio Laboral Revista Venezolana, 4(8). Obtenido de https://www.redalyc.org/articulo.oa?id=2190/219022148006

5. Hernández, I., Salazar, D., Rodríguez, M., \& S, F. (2010). Estrategia para integrar la gestión del capital humano a la gestión empresarial. Ingeniería Industrial, XXXI (3), 1-10. Obtenido de https://www.redalyc.org/pdf/3604/360433574002.pdf

6. Jimenez, C. (2018). Gestión del capital humano. Obtenido de Universidad De Alicante: https://www.unniun.com/la-importancia-del-capital-humano-cecilia-jimenez-masterdireccion-gestion-empresas-universidad-alicante-mde

7. León, E. (2013). Gestión del talento humano en las pequeñas y medianas empresas en el área Urbana de Retalhuleu. . UNIVERSIDAD RAFAEL LANDÍVAR. Obtenido de http://biblio3.url.edu.gt/Tesario/2013/05/57/De\%20Leon-Edy.pdf 
8. Los Recursos Humanos. (2019). La importancia del capital humano en las organizaciones. Obtenido de Los recursos humanos: https://www.losrecursoshumanos.com/laimportancia-del-capital-humano-en-las-organizaciones/

9. Luna, J. (2012). Influencia del capital humano para la competitividad de las pymes en el sector manufacturero de celaya, Guanajuato. Universidad de Celaya. Obtenido de http://www.eumed.net/tesis-doctorales/2013/jelc/jelc.pdf

10. Montoya, C., \& Boyero, M. (2016). El recurso humano como elemento fundamental para la gestión de calidad y la competitividad organizacional. Revista Científica "Visión de Futuro", 20(2), 1-20.

11. Reyes, J. (4 de Mayo de 2015). Gestión de Recursos humanos. Importancia objetivos. Obtenido de Escuela de Organización Industrial: https://www.eoi.es/blogs/mintecon/2015/05/04/gestion-de-recursos-humanosimportancia-y-objetivos/

12. Rojas, R., \& Vilchez, S. (2018). Gestión del talento humano y su relación con el desempeño laboral del personal del puesto de salud sagrado corazón de Jesús. Lima: Universidad Norbert Wiener.

13. Valencia, M. (2005). El capital humano, otro activo de su empresa. Entramado, 1(2), 2033. Obtenido de https://www.redalyc.org/pdf/2654/265420471004.pdf

14. Valentín, H. (2016). Gestión del Talento Humano y Desempeño Laboral del Personal de la Red de Salud Huaylas Sur. Lima: Universidad Inca de Garcilaso de las Vega. Obtenido de http://repositorio.uigv.edu.pe/handle/20.500.11818/2108

\section{References}

1. Alonso, C., Parra, R., \& Arronte, L. (2011). A human capital management system in emce based on labor competencies. Rev Ing Ind, XXXI (1). Retrieved from http://www.redalyc.org/pdf/3604/360433572003.pdf\&sa=U\&ved=2ahUKEwiE3on 
2. Big River. (January 12, 2018). The importance of human capital for success in business. Obtained from Human Resources System: http://sistemarecursoshumanos.com/laimportancia-los-objetivos-la-gestion-recursos-humanos/

3. Castillo, R. (2012). Development of human capital in organizations. . Mexico: Third $\begin{array}{llll}\text { Millennium } & \text { Network. } & \text { Obtained }\end{array}$ http://www.aliat.org.mx/LibrariesDigitales/economico_administrativo/Desarrollo_del_ca pital_humano_en_las_org.pdf

4. Del Canto, E. (2011). Human capital management, skills and knowledge society. Labor Observatory Revista Venezolana, 4 (8). Retrieved from https://www.redalyc.org/articulo.oa?id=2190/219022148006

5. Hernández, I., Salazar, D., Rodríguez, M., \& S, F. (2010). Strategy to integrate human capital management into business management. Industrial Engineering, XXXI (3), 1-10. Retrieved from https://www.redalyc.org/pdf/3604/360433574002.pdf

6. Jimenez, C. (2018). Human capital management. Obtained from Universidad De Alicante: https://www.unniun.com/la-importancia-del-capital-humano-cecilia-jimenez-masterdireccion-gestion-empresas-universidad-alicante-mde

7. León, E. (2013). Management of human talent in small and medium enterprises in the Urban area of Retalhuleu. . RAFAEL LANDÍVAR UNIVERSITY. Retrieved from http://biblio3.url.edu.gt/Tesario/2013/05/57/De\%20Leon-Edy.pdf

8. Human Resources. (2019). The importance of human capital in organizations. Obtained from Human resources: https://www.losrecursoshumanos.com/la-importancia-del-capitalhumano-en-las-organizaciones/

9. Luna, J. (2012). Influence of human capital for the competitiveness of SMEs in the manufacturing sector of Celaya, Guanajuato. University of Celaya. Retrieved from http://www.eumed.net/tesis-doctorales/2013/jelc/jelc.pdf 
10. Montoya, C., \& Boyero, M. (2016). Human resources as a fundamental element for quality management and organizational competitiveness. Scientific Magazine "Vision of the Future", 20 (2), 1-20.

11. Reyes, J. (May 4, 2015). Human resources management. Importance objectives. Obtained from Industrial Organization School: https://www.eoi.es/blogs/mintecon/2015/05/04/gestion-de-recursos-humanosimportancia-y-objetivos/

12. Rojas, R., \& Vilchez, S. (2018). Management of human talent and its relationship with the work performance of the staff of the Sacred Heart of Jesus health post. Lima: Norbert Wiener University.

13. Valencia, M. (2005). Human capital, another asset of your company. Lattice, 1 (2), 20-33. Retrieved from https://www.redalyc.org/pdf/2654/265420471004.pdf

14. Valentín, H. (2016). Human Talent Management and Labor Performance of the Health Network Staff Huaylas Sur. Lima: Inca University of Garcilaso de las Vega. Retrieved from http://repositorio.uigv.edu.pe/handle/20.500.11818/2108 